\title{
Mechanical and Chemical Root Preparation In Vitro: Efficiency of Plaque and Calculus Removal
}

\author{
Bradford M. Eschler* and John W. Rapley ${ }^{\dagger}$
}

THE AIM OF THIS INVESTIGATION was to compare the effectiveness of several different methods of root instrumentation by measuring and comparing the amount of residual stained material following treatment. Also, 2 different methods of quantitating residual stained material were compared. A total of 90 periodontally-involved teeth were extracted and randomly assigned to 1 of 8 treatment groups or to the untreated control group. Experimental treatments consisted of one or a combination of the following: Columbia 13-14 curet, P-10 ultrasonic instrument, diamond-coated P-10 ultrasonic instrument, or antiformin/citric acid chemical treatment. Selected samples were examined using light microscopy in order to determine the amount of cementum removed during root instrumentation. Residual stained material was quantitated using a method of grid-square analysis as well as by the use of photographs and a digitizing tablet. Following instrumentation, it was noted in histologic sections that the complete removal of cementum was rare, although all of the cementum was removed by some experimental treatments in some areas. All mechanical methods of root instrumentation were found to be essentially equal in effectiveness with respect to the removal of plaque and calculus. Chemical debridement alone was found to be ineffective. It was further noted that the grid-square method of analysis produced measurements that were 2 to 8 times higher than measurements produced by the digitizing tablet. $J$ Periodontol 1991; 62:755-760.

Key Words: Antiformin; tooth root; scaling; planing; dental plaque/prevention and control; dental calculus/prevention and control; ultrasonics/therapeutic use.

Bacterial plaque is recognized as the cause of periodontitis. ${ }^{1-6}$ Calculus, a secondary etiologic factor, facilitates plaque formation and retention by virtue of its tenacious attachment to the root surface. The root surface that is exposed by periodontal disease is also thought to contribute to the disease process by acting as a reservoir for bacterial substances that contribute to inflammation and loss of bone and attachment. ${ }^{7,8}$ The removal of plaque, calculus, and altered cementum by means of scaling and root planing is one fundamental procedure in periodontal treatment. ${ }^{9}$ In addition, various chemical treatments of the root surface have

\footnotetext{
*Currently, Department of Periodontics, Prevention, and Geriatrics, University of Michigan, Ann Arbor, MI; previously, Wilford Hall Medical Center/SGS, Lackland Air Force Base, TX.

${ }^{\dagger}$ Currently, Department of Periodontics, University of Missouri-Kansas City, School of Dentistry, Kansas City, MO; previously, Wilford Hall Medical Center/SGS, Lackland Air Force Base, TX.

The views expressed in this article are those of the authors and do not reflect the official policy of the Department of Defense or other departments of the United States Government.
}

been suggested as methods to detoxify the root surface or to demineralize the root surface in order to enhance new attachment formation. ${ }^{10-18}$

The ultimate objective of all root treatment procedures is to render the treated root surface biologically compatible for reattachment with host periodontal tissues. Biological compatibility is a term that is used without a precise scientific definition, but implies that root contamination by bacterial products has been reduced below some critical threshold, thereby facilitating repair and reattachment within the periodontium. All studies done to investigate the effects of root instrumentation are attempts to assess biological compatibility.

Clinical trials have directly assessed the biological compatibility of treated root surfaces and have demonstrated that periodontal treatment based upon root debridement results in improvement of periodontal health. ${ }^{19-23}$ Other studies have attempted to assess the effectiveness of root treatment procedures by measurement of stainable material left behind following treatment. ${ }^{24-26}$ Investigations of this type have 
shown that complete debridement of periodontally involved root surfaces is seldom achieved in the clinical setting.

In this investigation, the amounts of residual material on root surfaces that were treated in vitro by several different mechanical and/or chemical modalities were compared. While the nature of residual stained material is not addressed experimentally, the problem of overestimation of residual stained material is examined by comparing two different methods of measurement.

\section{MATERIALS AND METHODS}

\section{Collection of Experimental Samples}

Ninety periodontally involved teeth with at least $5 \mathrm{~mm}$ of interproximal probing depth were utilized from patients who required extractions as part of periodontal or prosthetic treatment. After informed consent was obtained, the gingival margin was inscribed on the tooth using a $1 / 4$ round bur in a high-speed handpiece at the line angles of the tooth. Following extraction, study teeth were rinsed with water for approximately 60 seconds and placed in normal saline.

\section{Experimental Treatment and Segregation of Samples}

Experimental samples were treated within 24 hours of collection. Bur marks made at the line angles demarcating the free gingival margin were connected to outline the entire gingival margin. Following this initial processing, each sample tooth was assigned to either 1 of the 8 experimental treatment groups or to the control group. Ten teeth were randomly assigned to each group. Mesial and distal root surfaces were instrumented and/or chemically treated from the facial line angle to the lingual line angle and from the coronally inscribed bur mark on the tooth to the coronal aspect of the tissue attachment (assessed by visual inspection). The 8 experimental groups were treated with 1 or more of 4 treatment modalities.

\section{Experimental Groups}

Group 1. Control group.

Group 2. The ultrasonic instrument ${ }^{\ddagger}$ was used with a P10 tip on its highest setting. Samples were instrumented to visual and tactile smoothness as assessed by a \#23 explorer.

Group 3. Teeth were scaled and root planed to visual and tactile smoothness using a sharp Columbia 13-14 curet that was resharpened. Tactile smoothness was assessed using a \#23 explorer.

Group 4. Same treatment as in Group 3. These root surfaces were then treated with antiformin solution for a period of 5 minutes, immersed in 5\% citric acid solution for 30 seconds, and then rinsed for 1 minute.

Group 5. An ultrasonic instrument ${ }^{\ddagger}$ was used with a diamond-coated P-10 tip on its highest setting. These root surfaces were treated until visibly free of accretions. Tactile smoothness was not obtainable with this instrument.

${ }^{\ddagger}$ Cavitron; Dentsply, York, PA.
Group 6. Same treatment as in Group 5; teeth were then treated with antiformin/citric acid as described in Group 4 above.

Group 7. Same treatment as in Group 5, then same treatment as Group 3.

Group 8. Same treatment as in Group 7, then antiformin/ citric acid treatment was carried out as described for Group 4.

Group 9. Samples in this group received the antiformincitric acid chemical treatment only.

Following treatment, each sample was divided buccolingually using a diamond disk and samples were again rinsed with water for 1 minute to remove grinding debris and the resulting halves were either stained and scored for residual plaque and calculus, or frozen in normal saline for use in a later, related study.

\section{Staining of Samples}

The fragment designated for staining was immersed in $1 \%$ methylene blue and after 2 minutes, each tooth fragment was rinsed in running water for 1 minute, and then fixed and stored in $10 \%$ formalin. The samples were refrigerated until scored. Generally, samples were read within 48 hours of treatment.

\section{Scoring of Residual Stained Material}

The amount of residual stained material was assessed using 2 different scoring methods. Control and treated surfaces were viewed at $10 \times$ magnification through a stereomicroscope fitted with a grid to divide the field into 100 equal parts. ${ }^{27}$ Each square (1/100 of grid) that contained any stained material was counted as 1 unit. The total number of squares for the entire root surface were then counted. The number of units containing residual stained material was divided by the total number of squares and reported as a percentage.

The second method of analysis utilized the Zeiss Interactive Data Analysis System (ZIDAS). The ZIDAS is a digitizing tablet system that allows the user to precisely measure the area of a figure by tracing the perimeter of that figure with a cursor and cross-hair system. Photographs of experimental samples were taken through the dissecting microscope at $10 \times$ magnification. Slides were projected and traced on $81 / 2^{\prime \prime} \times 11^{\prime \prime}$ sheets of paper; images were then traced using the ZIDAS. Parameters measured were the total area of the sample and the summed total of all of the residual stained areas within the sample. Residual stained material was reported as a percentage of the total stained area for each sample.

\section{Light Microscopy}

Two samples from each experimental group of root fragments that had been examined for residual stained material were randomly selected for examination by light microscopy. Samples were decalcified in a solution of sodium citrate/formic acid for 7 days. After decalcification, sectioned teeth were dehydrated using graded alcohols fol- 
Table 1. Group Means (percentages) and Standard Deviations for Grid and ZIDAS Data

\begin{tabular}{lrr}
\hline Group & \multicolumn{1}{c}{ Grid } & \multicolumn{1}{c}{ ZIDAS } \\
\hline 1 & $77.5 \pm 14.4$ & $45.8 \pm 24.5$ \\
2 & $19.3 \pm 17.1$ & $5.7 \pm 10.7$ \\
3 & $7.6 \pm 6.6$ & $1.0 \pm 1.2$ \\
4 & $6.6 \pm 6.5$ & $0.4 \pm 0.5$ \\
5 & $7.4 \pm 7.8$ & $0.9 \pm 0.6$ \\
6 & $5.9 \pm 6.5$ & $1.1 \pm 1.0$ \\
7 & $4.3 \pm 4.2$ & $0.9 \pm 0.7$ \\
8 & $9.7 \pm 10.0$ & $2.3 \pm 2.6$ \\
9 & $84.1 \pm 18.9$ & $41.6 \pm 17.4$ \\
\hline
\end{tabular}

$1=$ Untreated control.

$2=\mathrm{P}-10$ ultrasonic instrument.

$3=$ Curet

$4=$ Curet/antiformin-citric acid.

5 = Diamond-coated P-10 ultrasonic instrument.

6 = Diamond-coated P-10 ultrasonic instrument/antiformin-citric acid.

7 = Diamond-coated P-10 ultrasonic instrument/curet.

8 = Diamond-coated P-10 ultrasonic instrument/curet/antiformin-citric acid.

$9=$ Antiformin-citric acid.

lowed by 2 changes of xylene. Samples were embedded in paraffin and sectioned at $6 \mu \mathrm{m}$ in an occluso-apical dimension. Sections were stained using Harris's hematoxylin and eosin-Y solutions. Three sections from the center of each sample were examined for the presence of cementum on the treated root surface.

\section{Analysis of Data}

Statistical analyses were performed using the Statview 512 Software Package running on an Apple Macintosh Plus microcomputer. Data from the residual stained material portion of the investigation was analyzed using analysis of variance (ANOVA). Two data sets were analyzed separately; measurements obtained using the grid method were tested with ANOVA, as were measurements obtained using ZIDAS. Scheffe's F-test was used as the post hoc test. In addition, correlation between the data obtained using the grid method and ZIDAS was performed using Pearson's " $r$ " method of correlation.

Results from light microscopy were descriptive and did not require statistical analysis.

\section{RESULTS}

\section{Residual Stained Material}

Since stained surfaces were analyzed using the grid-square method of analysis and the ZIDAS, 2 separate sets of data were produced (Table 1). These 2 data sets were analyzed individually using ANOVA and Scheffe's F-test.

The mean percentage of residual stained material determined from the grid-square method of analysis ranged from $4.3 \%$ to $84.1 \%$ (Table 1). ANOVA disclosed significant differences between the means of the experimental groups at $P<0.01$. It should be noted that there were large standard deviations compared to the means and that the standard deviations of the different groups were not homogeneous.
Scheffe's F-test was used as the post hoc test to discriminate between the means. Using this post hoc test, all pairs of means were compared to one another. It was found that the mean for the untreated control group was similar to the mean for the antiformin/citric acid group. All of the groups that used mechanical methods of instrumentation were found to have significantly less residual stained material than the control group and the antiformin/citric acid group. Among the groups in which mechanical instrumentation had been used, only 1 pair of treatments was significantly different from one another; significantly more residual stained material remained following treatment with $\mathrm{P}-10$ ultrasonic instrument (Group 2) than remained after combined treatment with the diamond-coated ultrasonic instrument and curet (Group 7). All other pairings among the groups that used mechanical instrumentation were statistically similar to one another.

The mean percentage of residual stained material determined by ZIDAS measurements ranged from $0.4 \%$ to $45.8 \%$ (Table 1). On the basis of ANOVA, the null hypothesis that there was no difference between the means of the various groups was rejected $(P<0.01)$. When Scheffe's F-test was used to compare the means of the various groups to one another $(P<0.01)$, the control group (Group 1) and the antiformin/citric acid group (Group 9) were found to be similar. Significantly more residual stained material was left behind in these groups (Groups 1,9) than in the groups in which mechanical instrumentation was used (Groups 2 to 8). Among the groups that had utilized mechanical instrumentation, there were no statistically significant differences.

When the grid-square data set was compared to the ZIDAS data set, it was found that the grid-square measurements were 2 to 8 times higher than the ZIDAS measurements. Despite these numerical differences, a high correlation was found between the two methods $(\mathrm{r}=0.875$, $P<0.001$ ) (Fig. 1).

\section{Description of Light Microscopic Sections}

A summary of findings from the light microscopic examination is depicted in Table 2. Two samples from each experimental group were processed for light microscopy. Despite processing problems, sections were obtained for each experimental group. Three serial, longitudinal, buccolingual sections were taken from mid-root at $50 \mu \mathrm{m}$ intervals in order to determine the extent of cementum removal. In all specimens, the notch placed at the free gingival margin was clearly visible. In several specimens, remnants of the connective tissue attachment could be seen, but in general, it was difficult to determine the apical extent of root instrumentation.

Cementum was observed in all sections of all specimens. However, cementum was removed in the more coronal portions of sections from 4 experimental groups. Cementum was removed from specimens instrumented by the P-10 ultrasonic instrument and for a considerable distance in the 


\section{CORRELATION: GRID VS. ZIDAS}

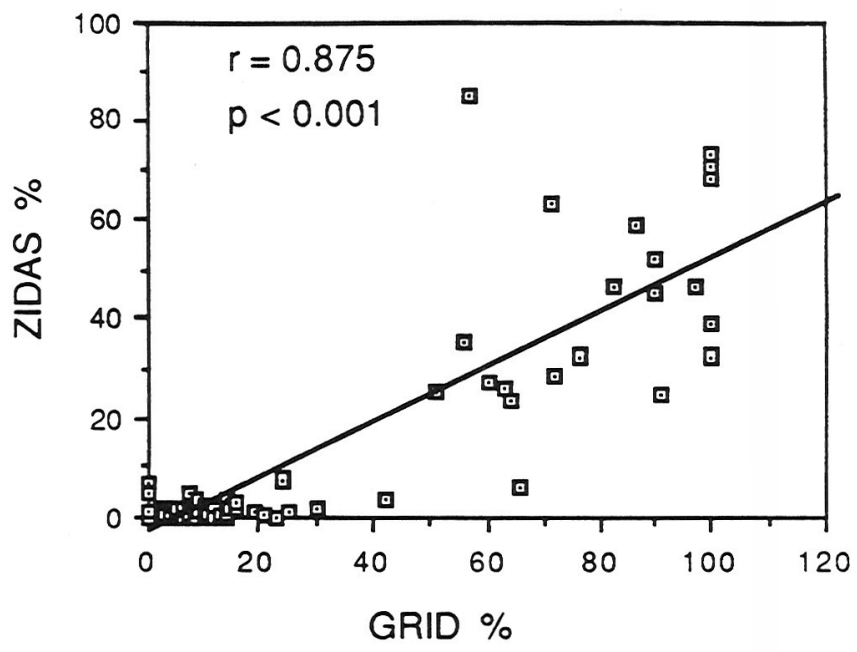

Figure 1. Scatter plot for grid vs. ZIDAS. Although the values for the grid were 2 to 8 times higher than ZIDAS measurement, there was a high correlation between the two sets of measurements $(r=0.875, \mathrm{P}<0.001)$.

Table 2. Summary of Light Microscopic Finding

\begin{tabular}{lc}
\hline Group & $\begin{array}{c}\text { Total Cementum } \\
\text { Removal }\end{array}$ \\
\hline $1=$ Untreated control. & No \\
$2=$ P-10 ultrasonic instrument. & Yes \\
$3=$ Curet. & No \\
$4=$ Curet/antiformin-citric acid. & No \\
$5=$ Diamond-coated P-10 ultrasonic instrument. & Yes \\
$6=$ Diamond-coated P-10 ultrasonic instrument/ & No \\
antiformin-citric acid. & \\
$7=$ Diamond-coated P-10 ultrasonic instrument/ \\
curet. \\
$8=$ Diamond-coated P-10 ultrasonic instrument/ \\
curet/antiformin-citric acid. \\
$9=$ Antiformin-citric acid. & Yes \\
\end{tabular}

*"Yes" or "No" refers to whether or not all cementum was removed from a portion of the root surface.

area below the coronal notch. The area of cementum removal terminated abruptly, leaving a step-like boundary between the dentin and the remaining cementum. Samples treated with a combination of diamond-coated P-10 ultrasonic tip, curet, and antiformin/citric acid also demonstrated complete removal of cementum. In contrast to the $\mathrm{P}-10$ samples, the sections from samples treated with this combination treatment had a smooth beveled transition from dentin to remaining cementum. One of the samples treated with a combination of the diamond-coated P-10 tip and antiformin/citric acid showed a small area of complete cementum removal just subjacent to the notch. A similar finding was noted for one sample treated by the diamond-coated $\mathrm{P}-10$ instrument alone.

In the remainder of the instrumented, chemically-treated and control samples, either cementum was not removed at all or only a partial thickness was removed.

\section{DISCUSSION}

When comparing the amount of tooth structure removed from treated root surfaces in this study to effects documented in other studies, methodological differences between experiments must be taken into account. In this investigation, root surfaces were treated to clinical endpoints. Test surfaces were treated until free of visual accretions and smooth to exploration. Using these parameters, the complete removal of cementum in histologic sections was found to be an uncommon occurrence. In general, the only samples from which cementum was completely removed were those treated with either the P-10 or the diamond-coated P-10 when used in combination with other instruments. In the case of the P-10 tip, it seems likely that cementum was not removed by planing per se, but by means of irregular loss of cementum due to the hammering action of the ultrasonic tip. ${ }^{28,29}$ This type of irregular cementum loss is supported by the abrupt, step-like transition from dentin to cementum observed in the P-10 light microscopic sections. In contrast to the step-like transition from dentin to cementum observed in the P-10 sections, a gradual transition from dentin to cementum was seen in histologic sections from samples in which the diamond-coated P-10 tip is a planing instrument. On the basis of light microscopy, the diamond-coated P-10 ultrasonic tip was shown to be capable of removing cementum in most cases, especially when used in conjunction with other methods of debridement.

Root surface damage that occurs when the ultrasonic instrument is used is related to pressure of application, time of application, and power setting. Differing opinions regarding the amount of root surface damage caused by the ultrasonic instrument may be found in the literature. In one study, it has been reported that the ultrasonic instrument was as effective as curets in removing accretions, but caused far less root surface damage. ${ }^{30}$ Other authors have found that the ultrasonic instrument causes a significant amount of root surface alteration. ${ }^{28,29,31,32}$ It may be speculated that the power setting of the instrument may have been responsible for the varied results reported in these studies. In this investigation, as well as in others, ${ }^{28,32}$ the ultrasonic instrument was used on its high setting. It is likely that the use of this instrument on its high setting is reponsible for the loss of tooth structure. One study ${ }^{29}$ used the ultrasonic instrument on its medium setting and found less root surface damage than when the instrument was used on the high setting.

Results from this study have shown that it is possible to effectively remove root surface accretions under ideal conditions of visibility and access. Because of the methodological differences between this investigation and other studies of residual stained material, direct comparisons cannot be made between this study and other experiments that have used this parameter. The intent of this investigation was to compare methods of root preparation without the limitations of access imposed by the clinical situation. Under the conditions of the test, it was found that all mechanical methods of instrumentation were essentially equal. Furthermore, the 
use of antiformin/citric acid alone is ineffective in the removal of root surface accretions.

When the traditional grid-square method of measurement was tested against the ZIDAS digitizing tablet method of quantitating residual stained material, it was found that the grid method produced measurements that were 2 to 8 times greater than measurements made using the ZIDAS on the same material. The grid method as described by Rabbani et al. ${ }^{22}$ and used by several other investigators scores as a positive any grid square that contains residual stained material without regard to the amount of actual surface area covered by the residual material within the square. ${ }^{24,27,28}$ Therefore, it was hypothesized that this method of estimation might produce values for residual stained material that are greater than values produced by the digitizing tablet which measures area directly from tracings made from projected images. This hypothesis was borne out in the data when both methods of measurement were compared. Although there were numerical differences between the data sets, there was a high correlation between the 2 methods of measurement, implying that both techniques measure the same phenomenon. From a quantitative standpoint, the data generated by ZIDAS in this investigation indicate that the outlook for effective root instrumentation may not be as pessimistic as previously reported..$^{24,27,28}$ It seems likely that, if previous studies had used a more nearly representative technique of measurement, such as a digitizing tablet, reported percentages for residual material might have been much lower. However, some caution must be exercised in making this interpretation due to methodological differences between the current in vitro study and previous in vivo studies.

A problem that is encountered in studies of residual stained material is the lack of specificity of the stained material and the resulting difficulty in interpretation. It is difficult to decide whether or not the material that is stained actually represents plaque and calculus. For example, several samples stained a diffuse, light blue that could be misinterpreted as residual plaque and calculus. It has been shown that cementum and superficial layers of dentin are capable of taking up stain and confounding the interpretation of stained material. ${ }^{33}$ Others have demonstrated that the majority of the stained material on a recently instrumented and extracted root surface consists of instrumentation debris, fibrin, and retained pellicle, rather than plaque. ${ }^{15}$ The current investigation clearly shows that over estimation of residual stained material occurs when the grid-square method of analysis is used. Because of this problem and the possible lack of biological significance of residual stained material reported by other authors, the value of this parameter as an index of the effectiveness of root treatment procedures must be questioned.

\section{SUMMARY AND CONCLUSIONS}

Two conclusions may be drawn from this study. Under conditions of unlimited access, all mechanical methods of root instrumentation were essentially equal. One may conclude from this observation that access is potentially the critical element in success in root instrumentation since all methods of instrumentation were essentially equal under conditions of unlimited access. Secondly, caution must be used in interpreting results from studies of residual stained material since the outcome of such studies is affected by the method of measurement employed. On the basis of the current data, it seems likely that those studies have used the grid-square method of analysis may be particularly prone to the problem of overestimation.

\section{Acknowledgment}

This study was funded by the United States Air Force through the Wilford Hall Medical Center/SGS, Lackland Air Force Base, TX 78236.

\section{REFERENCES}

1. Löe H, Theilade E, Jensen S, Schiott C. Experimental gingivitis in man. III. The influence of antibiotics on gingival plaque development. $J$ Periodont Res 1967;2:282-289.

2. Page R, Schroeder H. Pathogenesis of inflammatory periodontal disease-A summary of current work. Lab Invest 1976; 33:235-249.

3. Theilade $E$, Wright $W$, Jensen $S$, Löe $H$. Experimental gingivitis in man. II. A longitudinal clinical and bacteriologic investigation. $J$ Periodont Res 1966;1:1-13.

4. Löe H. Human research model for the production and prevention of gingivitis. J Dent Res 1971:50:256-264.

5. Socransky S. Relationship of bacteria to the etiology of periodontal disease. J Dent Res 1970;49:203-222.

6. Löe H, Theilade E, Jensen S. Experimental gingivitis in man. J Periodontol 1965;36:177-187.

7. Jones $\mathrm{W}, \mathrm{O}$ 'Leary $\mathrm{T}$. The effectiveness of in vivo root planing in removing bacterial endotoxin from roots of periodontally involved teeth. J Periodontol 1978;49:337-342.

8. Daly C, Seymour J, Kieser J. Bacterial endotoxin: A role in chronic inflammatory periodontal disease. J Oral Pathol 1980;9:1-15.

9. Lang N. Indications and rationale for nonsurgical periodontal therapy. Inter Dent $J$ 1983;33:127-136.

10. Register A, Burdick F. Accelerated reattachment with cementogenesis to dentin, demineralized in situ. I. Optimum range. $J$ Periodontol 1975;46:646-655.

11. Common J, McFall W. The effects of citric acid on attachment of laterally positioned flaps. J Periodontol 1983;54:9-18.

12. Sarbinoff J, O'Leary T, Miller C. The comparative effectiveness of various agents in detoxifying diseased root surfaces. $J$ Periodontol $1983 ; 54: 77-80$.

13. Albair W, Cobb C, Killoy W. Connective tissue attachment to periodontally diseased roots after citric acid demineralization. $J$ Periodontol 1982;53:515-526.

14. Lopez N. Connective tissue to periodontally diseased roots, planed and conditioned with citric acid implants into the oral mucosa. $J$ Periodontol 1984;55:381-390.

15. Smith B, Mason W, Morrison E, Caffesse R. The effectiveness of citric acid as an adjunct to surgical reattachment procedures in humans. J Clin Periodontol 1986;13:701-708.

16. Sterrett J, Murphy H. Citric acid burnishing of dentinal root surfaces. A scanning electron microscopy report. J Clin Periodontol 1989;16:98104.

17. Lasho D, O'Leary T, Kafrawy A. A scanning electron microscope study of the effects of various agents on instrumented periodontally involved root surfaces. $J$ Periodontol 1983;54:210-220.

18. Garrett J, Crigger M, Egelberg J. Effects of citric acid on diseased root surfaces. J Periodont Res 1978;13:155-163.

19. Loos B, Nylund K, Claffey N, Egelberg J. Clinical effects of root 
debridement in molar and non-molar teeth. A two-year follow-up. $J$ Clin Periodontol 1989;16:498-504.

20. Tagge D, O'Leary T, El-Kafrawy A. The clinical and histologic response of periodontal pockets to root planing and oral hygiene. $J$ Periodontol 1975; 46:527-533.

21. Badersten A, Nilveus R, Egelberg J. Effect of non-surgical periodontal therapy. II. Severely advanced periodontitis. J Clin Periodontol 1984;11:63-76.

22. Morrison E, Ramfjord S, Hill R. Short-term effects of initial, nonsurgical periodontal treatment (hygienic phase). J Clin Periodontol 1980;7:199-211.

23. Cercek J, Kiger R, Garrett S, Egelberg J. Relative effects of plaque control and instrumentation on the clinical parameters of human periodontal disease. J Clin Periodontol 1983;10:46-56.

24. Caffesse R, Sweeney P, Smith B. Scaling and root planing with and without periodontal flap surgery. J Clin Periodontol 1986;13:205210.

25. Fleisher H, Mellonig J, Brayer W, Gray J, Barnett J. Scaling and root planing efficacy in multirooted teeth. $J$ Periodontol 1989;60:402409.

26. Wylam J, Mills M, Moskowicz D. Effectiveness of scaling on molar teeth-surgical vs. non-surgical approach. $J$ Dent Res 1986;65:(Spec. Issue) 270 (Abstr. 911).
27. Rabbani G, Ash M, Caffesse R. The effectiveness of subgingival scaling and root planing in calculus removal. $J$ Periodontol 1981;52:119-123.

28. Breininger D. O'Leary T, Blumenshine R. Comparative effectiveness of ultrasonic and hand scaling for the removal of subgingival plaque and calculus. $J$ Periodontol 1987;58:9-18.

29. Lie T, Leknes K. Evaluation of the effect on root surfaces of air turbine scalers and ultrasonic instrumentation. J Periodontol 1985;56:522-531.

30. Jones S, Lozdan J, Boyde A. Tooth surfaces treated in situ with periodontal instruments: Scanning electron microscopic studies. Brit Dent J 1972;132:57-64.

31. Allen E, Rhoads R. Effects of high speed periodontal instruments on tooth surfaces. J Periodontol 1963;34:352-356.

32. Wilkinson R, Maybury J. Scanning electron microscopy of the root surface following instrumentation. J Periodontol 1973;44:559-563.

33. Poole S, Kieser J, Boyde A, Jones S, Reid S. Recent developments in SEM shed light on root planing. J Dent Res 1984;63:497.

Send reprint requests to: Dr. John W. Rapley, Department of Periodontics, University of Missouri-Kansas City, School of Dentistry, 650 East 25th St., Kansas City, MO 64108.

Accepted for publication July 29, 1991. 